\title{
Hydrodynamics of self-propelled hard rods
}

\author{
Aparna Baskaran and M. Cristina Marchetti \\ Physics Department, Syracuse University, Syracuse, New York 13244, USA
}

(Received 17 August 2007; published 25 January 2008)

\begin{abstract}
Motivated by recent simulations and by experiments on aggregation of gliding bacteria, we study a model of the collective dynamics of self-propelled hard rods on a substrate in two dimensions. The rods have finite size, interact via excluded volume, and their dynamics is overdamped by the interaction with the substrate. Starting from a microscopic model with nonthermal noise sources, a continuum description of the system is derived. The hydrodynamic equations are then used to characterize the possible steady states of the systems and their stability as a function of the particles packing fraction and the speed of self-propulsion.
\end{abstract}

DOI: 10.1103/PhysRevE.77.011920

PACS number(s): 87.18.Ed, 47.54. $-\mathrm{r}, 05.65 .+\mathrm{b}$

\section{INTRODUCTION}

Self-propelled particles draw energy from internal or external sources and dissipate this energy by moving through the medium they inhabit. Such a definition encompasses a wide class of systems such as fish schools, bacterial colonies, and monolayers of vibrated granular rods. In all of these the energy input that maintains the system out of equilibrium is on each unit, rather than at the boundaries as in more conventional nonequilibrium situations [1]. A striking phenomenon exhibited by these systems is flocking, the emergence of a coherently moving body of self-propelled entities of size much larger than the length scale of the interparticle interaction.

Extensive theoretical effort has been devoted to understanding these nonequilibrium systems. Two distinct approaches have been used. First, starting with the seminal work by Vicsek [2], a number of numerical studies of simplified rule-based model systems have been carried out. A second approach has been to write down generic continuum theories based on symmetry considerations [3,4]. These methods are well developed for equilibrium systems, where general properties such as the fluctuation-dissipation theorem yield strong constraints on the parameters of the dynamical model $[5,6]$. They are also very useful for nonequilibrium systems, although in this case the parameters in the equations remain largely undetermined. Both approaches have exposed the dramatically different nature of order-disorder transitions and of the fluctuations in the various phases exhibited by self-propelled systems when compared to their equilibrium counterparts. Collections of self-propelled units can exhibit long-range order in two dimensions [3], in sharp contrast to equilibrium systems, where the continuous rotational symmetry cannot spontaneously be broken in systems with shortrange interactions [7]. Recent numerical work has shown that the Vicsek model and other closely related models with polar aligning interactions exhibit a first order (discontinuous) transition from a disordered to an ordered state in two dimensions when the density and the noise amplitude in the system are varied [8]. On the other hand, when this model is modified to induce strictly nematic order in the system, the phase transition becomes continuous [18]. In addition, large number fluctuations have been predicted and observed in the homogeneous states of these driven systems [9-11].
Nonequilibrium statistical mechanics is a powerful tool that can be brought to bear to describe this rich class of systems. One class of questions pertains to the identification of the underlying collective mechanisms that give rise to emergent behavior. Comparison of theoretical and numerical studies of model systems can provide insight into the physical origin of the various phenomena captured by the generic continuum theories. The second class of questions is associated with identifying minimal microscopic models capable of accounting for a given observed phenomenon. This is important when trying to generalize concepts developed in the context of animal group behavior to artificial systems considered in collective robotics [12]. An example of this approach is the recent work by Bertin et al. [13] who used nonequilibrium statistical mechanics to derive continuum hydrodynamic equations for the polar Vicsek model, whose coefficients are given explicitly in terms of parameters of the microscopic model.

In this work, we study the collective behavior of selfpropelled hard rods with excluded volume interactions, moving on a substrate in two dimensions. Two aspects distinguish this work from previous ones [13,14]. First, we provide the first theoretical discussion of the effect of finite size and shape of the self-propelled units. Secondly, we derive the hydrodynamic theory from a specific microscopic model. Part of our motivation comes from recent simulations of this system by Peruani et al. [15]. We consider a minimal model, with the aim of identifying the simplest physical mechanisms that can lead to organization on macroscopic scales in self-propelled systems with short-range interactions. With this scope in mind, no interaction beyond the physical excluded volume is included. This allows us to investigate the nature of entropic ordering in the presence of self-propulsion. The nonequilibrium statistical mechanics for this model is developed systematically and results in coarsegrained hydrodynamic equations for the slow variables of the system. The macroscopic equations are then used to characterize the homogeneous steady states and their stability as a function of particle density and self-propulsion velocity. Several new results are obtained. First, we show that apolar excluded volume interactions are not sufficient to yield bulk polar order of self-propelled rods on a substrate [16]. The only homogeneous states of the system are isotropic and nematic. Secondly, we find that the isotropic state can support novel propagating waves, as reported in experiments on 
contractile epithelial cells [17]. Thirdly, we demonstrate that the nematic state is unstable above a critical self-propulsion velocity, in agreement with simulations $[11,15]$. Our work provides a microscopic basis for some of the phenomena predicted in active nematics via generic continuum theories $[10]$ and observed in simulations [15,18,19].

A real system that may be described by our minimal model is myxobacteria on a substrate under starvation conditions. These bacteria are rodlike in shape, with an aspect ratio of about 7 , and retain their shape under movement. Under starvation conditions the $C$-signaling mechanism in these bacteria that give rise to reversal of the direction of motion is suppressed and alignment is thought to be brought about by steric interactions [20]. Our model is also relevant to collections of melanocytes and fibroblasts that are apolar in nature and exhibit nematic, but not polar order [21].

The layout of the paper is as follows. First, the microscopic model is introduced and the nonequilibrium statistical mechanics developed. In a low density approximation, this is described by a Smoluchowski equation. From this starting point, the hydrodynamic equations associated with the slow variables of the system, namely, the density, polarization (related to the self-propulsion flow field), and nematic order parameter, are derived. Next, the homogeneous steady states are identified and their linear stability with respect to spatial fluctuations characterized. This analysis is used to interpret the physical mechanism behind the emergence of inhomogeneities in the nematic state observed in the numerical work of Ref. [15] and the large number fluctuations predicted in [10].

\section{MICROSCOPIC MODEL}

As a minimal physical model for a system of selfpropelled particles, we consider a collection of rigid rods of length $\ell \gg b$, with $b$ the rod diameter, moving on a substrate in two dimensions (2D). Each rod is "self-propelled" in that it has a force $F$ acting on its center of mass and oriented along its long axis. The rods interact only via excluded volume interaction and their dynamics is overdamped. The exchange of momentum with the substrate due to friction provides the physical mechanism that results in all the rods on average having a constant velocity of magnitude $v_{0}$ along their long axis. At high packing fractions, the excluded volume interaction gives rise to orientational order, as in passive nematic liquid crystals.

Note that a flock of self-propelled particles is characterized by three properties: (1) centering - the ability of a collection of such particles to stay together, (2) velocity matching - all the particles are on average characterized by the same velocity, and (3) alignment - the particles are asymmetric and exhibit ordered states with either nematic or polar symmetry [22]. In our model, the rods have no attractive interactions and hence will not form a cohesive flock. We assume that the system is at a fixed homogeneous density $\rho_{0}$, which is maintained by confinement at the boundary of the system. Alignment arises from the excluded volume interaction. As noted above, the force $F$ together with friction from the substrate gives rise to velocity matching among the par- ticles. Hence the minimal model considered here has the necessary ingredients to study the collective behavior of selfpropelled particles.

Each rod is characterized by the position $\mathbf{r}_{i}$ of its center of mass and its orientation with respect to an external axis, described by a unit vector $\hat{\mathbf{u}}_{i}=\left(\cos \theta_{i}, \sin \theta_{i}\right)$ directed along the axis of the rod. The microscopic dynamics of the $i$ th rod is controlled by coupled Langevin equations,

$$
\begin{gathered}
\frac{\partial r_{i \alpha}}{\partial t}=-D_{\alpha \beta}^{i} \sum_{j} \frac{\partial}{\partial r_{i \beta}} V_{e x}\left(\mathbf{r}_{i j}, \theta_{i}, \theta_{j}\right)+v_{0} \hat{u}_{i \alpha}+\eta_{i \alpha}(t), \\
\frac{\partial \theta_{i}}{\partial t}=-D_{R} \sum_{j} \frac{\partial}{\partial \theta_{i}} V_{e x}\left(\mathbf{r}_{i j}, \theta_{i}, \theta_{j}\right)+\eta_{i}^{R}(t),
\end{gathered}
$$

where Greek indices denote Cartesian components and $\mathbf{r}_{i j}$ $=\mathbf{r}_{i}-\mathbf{r}_{j}$. The self-propulsion velocity of magnitude $v_{0}$ is directed along the long axis of the rod. We choose coordinates so that the $z$ axis is normal to the plane of the substrate. The excluded volume interaction $V_{e x}\left(r_{i j}, \theta_{i}, \theta_{j}\right)$ has the Onsager form, with

$$
\begin{aligned}
V_{e x}\left(\mathbf{r}_{i j}, \theta_{i}, \theta_{j}\right) & =1 & & \text { if particles } i, j \text { intersect } \\
& =0 & & \text { otherwise. }
\end{aligned}
$$

This effective interaction can be derived from the collision rules that govern the dynamics of $2 \mathrm{D}$ hard rods and represents a very good approximation to the true momentum exchange in the overdamped limit [23]. The diffusion tensor $D_{\alpha \beta}^{i}$ and the rotational diffusion rate $D_{R}$ are determined by the energy scale associated with the excluded volume interaction and the translational and rotational damping provided by the substrate. Finally, $\eta_{i \alpha}$ and $\eta_{i}^{R}$ are white noise sources with correlations

$$
\begin{gathered}
\left\langle\eta_{i \alpha}(t) \eta_{j \beta}\left(t^{\prime}\right)\right\rangle=\widetilde{D}_{\alpha \beta}^{i} \delta_{i j} \delta\left(t-t^{\prime}\right), \\
\left\langle\eta_{i}^{R}(t) \eta_{j}^{R}(t)\right\rangle=\widetilde{D}_{R} \delta_{i j} \delta\left(t-t^{\prime}\right) .
\end{gathered}
$$

For systems in thermal equilibrium the fluctuationdissipation theorem requires that the amplitudes of the correlation of the thermal noise be identical to the diffusive parameters controlling the excluded volume interaction, i.e., $\widetilde{D}_{\alpha \beta}^{i}=D_{\alpha \beta}^{i}$ and $\widetilde{D}_{R}=D_{R}$. This identification does not hold for nonequilibrium systems such as the one considered here, where the noise has nonthermal contributions. For selfpropelled suspensions noise arises in general from thermal Brownian motion, hydrodynamic interactions among the self-propelled units, and intrinsic fluctuations in the activity of each self-propelled unit. Brownian noise is expected to be very small for particles such as bacteria of size larger than a few microns. The other two sources of noise are intrinsically nonthermal and will generally not obey a fluctuationdissipation theorem. It can be shown, however, that the equality still holds for a dilute solution in the regime of small self-propulsion velocities [23]. When the self-propulsion becomes large the relationship between these diffusion constants is complicated. In the subsequent analysis, the emergent physics of the system is independent of these details. 
Therefore for simplicity we assume the noise amplitudes are simply given by their equilibrium counterparts, with

$$
\widetilde{D}_{\alpha \beta}^{i}=D_{\alpha \beta}^{i}=D_{\|} \hat{u}_{i \alpha} \hat{u}_{i \beta}+D_{\perp}\left(\delta_{\alpha \beta}-\hat{u}_{i \alpha} \hat{u}_{i \beta}\right) .
$$

For long thin rods the longitudinal and transverse diffusion constants $D_{\|}$and $D_{\perp}$ are simply related as $D_{\|}=2 D_{\perp} \equiv 2 D_{0}$, with $D_{0}=k_{B} T / \zeta$ for a single rod at low density where $T$ is the temperature and $\zeta$ is a material dependent friction constant characterizing the interaction of the rod and substrate. A similar approximation is used for the rotational noise amplitude, with

$$
\tilde{D}_{R}=D_{R} \simeq \frac{6 D_{0}}{\ell^{2}}
$$

where the last equality holds for infinitely thin rods at low density.

The noise averaged statistical mechanics of a system of overdamped Langevin equations is given by a Smoluchowski equation for the $N$-particle phase space density. In the low density limit, when two-particle correlations can be neglected, this results in an effective mean-field Smoluchowski equation of the familiar form for the one-particle probability density $c(\mathbf{r}, \theta, t)$ representing the density of rods with center of mass at $\mathbf{r}$ and orientation $\theta$ at time $t$. Its dynamics is governed by the equation

$$
\frac{\partial c(\mathbf{r}, \theta, t)}{\partial t}=-\nabla \cdot \mathbf{J}^{T}-\frac{\partial}{\partial \theta} J^{R},
$$

with translational and rotational currents

$$
\begin{gathered}
J_{\alpha}^{T}=-D_{\alpha \beta} c \frac{\partial V(\mathbf{r}, \theta)}{\partial r_{\beta}}+v_{0} \hat{u}_{\alpha} c-D_{\alpha \beta} \frac{\partial c}{\partial r_{\beta}}, \\
J^{R}=-D_{R} c \frac{\partial V(\mathbf{r}, \theta)}{\partial \theta}-D_{R} \frac{\partial c}{\partial \theta} .
\end{gathered}
$$

In Eqs. (9) and (10) the two-particle Onsager interaction defined in Eq. (3) enters in its mean-field form

$$
\begin{aligned}
V\left(\mathbf{r}_{1}, \theta_{1}\right) & =\int d \mathbf{r}_{2} \int d \theta_{2} V_{e x}\left(\mathbf{r}_{12}, \theta_{1}, \theta_{2}\right) c\left(\mathbf{r}_{2}, \theta_{2}, t\right) \\
& =\int d \boldsymbol{\xi} \int d \theta_{2}\left|\hat{\mathbf{u}}_{1} \times \hat{\mathbf{u}}_{2}\right| c\left(\mathbf{r}_{1}+\boldsymbol{\xi}, \theta_{2}, t\right),
\end{aligned}
$$

where $\boldsymbol{\xi}=\mathbf{r}_{2}-\mathbf{r}_{1} \simeq s_{1} \hat{\mathbf{u}}_{1}-s_{2} \hat{\mathbf{u}}_{2}$ and $-\ell / 2 \leqslant s_{i} \leqslant \ell / 2$ parametrizes the position along the $i$ th rod measured from its center of mass. As shown in Fig. 1, this is a measure of the area excluded by a rod at a point $\mathbf{r}_{1}$, oriented in the direction $\theta_{1}$ to all other rods in the system.

We emphasize that the self-propulsion mechanism enters the Langevin equations (1) and (2) only in the form of a center of mass force. Therefore it gives rise to no microscopic torques. This is reflected in the Smoluchowski equation above in the fact that the rotational fluxes in Eq. (10) contain no information about the self-propulsion. The velocity $v_{0}$ enters only the translational flux as an additional mass flux along the orientation of the rod. In the absence of selfpropulsion $\left(v_{0}=0\right)$, the microscopic equations and hence the

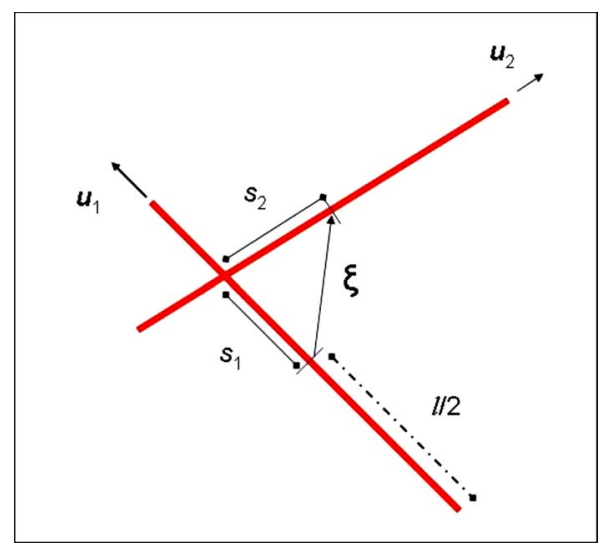

FIG. 1. (Color online) Geometry of overlap between two rods of length $\ell$. Here $\boldsymbol{\xi}=\mathbf{r}_{2}-\mathbf{r}_{1}$ is the separation of the centers of mass of the two rods, $s_{1}$ and $s_{2}$ parametrize the position along the rod relative to the center of mass, with $-\ell / 2 \leqslant s_{i} \leqslant \ell / 2$, for $i=1,2$. Finally, $\hat{\mathbf{u}}_{i}$ are the unit vectors directed along the rods' axes.

Smoluchowski equation are both invariant when $\hat{\mathbf{u}}_{i} \rightarrow-\hat{\mathbf{u}}_{i}$ for all rods and the system cannot exhibit a macroscopic polar state. When $v_{0} \neq 0$, this symmetry is broken at the level of the Langevin equations. One might then expect that the system can order in a uniform polar state. But since bulk states are controlled entirely by the rotational fluxes which still possess the nematic symmetry, such a homogeneous polar state cannot exist. This is not the consequence of any of the approximations made to obtain the Smoluchowski equation, but is a true property of the nonequilibrium model system described by the microscopic dynamics given in Eqs. (1) and (2). The self-propulsion term does, however, affect the fluctuations in the systems, as described in the next section.

\section{HYDRODYNAMIC EQUATIONS}

In this section we obtain coarse-grained equations appropriate for describing the dynamics of the system on length scales large compared to the length of the rods and time scales long compared to the microscopic diffusion times in the Langevin equations. In this limit we expect that the dynamics will be controlled by the conserved densities and the fields associated with possible broken symmetries. For an overdamped system of the type considered here the only conserved quantity is the concentration of rods. It can be defined as the zeroth moment of the probability distribution $c(\mathbf{r}, \hat{\mathbf{u}}, t)$,

$$
\rho(\mathbf{r}, t)=\int d \hat{\mathbf{u}} c(\mathbf{r}, \hat{\mathbf{u}}, t) .
$$

Both polar and nematic order are, in principle, possible in a gas of self-propelled rods. These can be described by a polarization vector $\mathbf{P}(\mathbf{r}, t)$ and the nematic alignment tensor $Q_{\alpha \beta}(\mathbf{r}, t)$ defined as first and second moments, respectively, of $c(\mathbf{r}, \hat{\mathbf{u}}, t)$,

$$
\rho(\mathbf{r}, t) \mathbf{P}(\mathbf{r}, t)=\int d \hat{\mathbf{u}} \hat{\mathbf{u}} c(\mathbf{r}, \hat{\mathbf{u}}, t),
$$




$$
\rho(\mathbf{r}, t) Q_{\alpha \beta}(\mathbf{r}, t)=\int d \hat{\mathbf{u}}\left(\hat{u}_{\alpha} \hat{u}_{\beta}-\frac{1}{2} \delta_{\alpha \beta}\right) c(\mathbf{r}, \hat{\mathbf{u}}, t) .
$$

Since each rod has a self-propulsion velocity $v_{0} \hat{\mathbf{u}}$, the polarization is also proportional to the self-propulsion flow field.

The equations for these continuum fields are obtained by taking the corresponding moments of the Smoluchowski equation (8) and have the form

$$
\begin{gathered}
\partial_{t} \rho=-\nabla \cdot \mathbf{J}, \\
\partial_{t}\left(\rho P_{\alpha}\right)=-\frac{\partial}{\partial r_{\beta}} J_{\alpha \beta}-R_{\alpha}, \\
\partial_{t}\left(\rho Q_{\alpha \beta}\right)=-\frac{\partial}{\partial r_{\gamma}} J_{\alpha \beta \gamma}-R_{\alpha \beta},
\end{gathered}
$$

where

$$
\left(\begin{array}{c}
J_{\gamma} \\
J_{\alpha \gamma} \\
J_{\alpha \beta \gamma}
\end{array}\right)=\int d \hat{\mathbf{u}}\left(\begin{array}{c}
1 \\
\hat{u}_{\alpha} \\
\left.\hat{u}_{\alpha} \hat{u}_{\beta}-\frac{1}{2} \delta_{\alpha \beta}\right)
\end{array}\right) J_{\gamma}^{T},
$$

and

$$
\left(\begin{array}{c}
R_{\alpha} \\
R_{\alpha \beta}
\end{array}\right)=\int d \hat{\mathbf{u}}\left(\left(\begin{array}{c}
\hat{u}_{\alpha} \\
\hat{u}_{\alpha} \hat{u}_{\beta}-\frac{1}{2} \delta_{\alpha \beta}
\end{array}\right)\right) \frac{\partial J^{R}}{\partial \theta},
$$

with the fluxes $\mathbf{J}^{T}$ and $J^{R}$ as given in Eqs. (9) and (10). In order to obtain a closed set of hydrodynamic equations, we need to evaluate the constitutive equations for the fluxes on the right-hand side of Eqs. (15)-(17). This requires various approximations. First, since we are interested in a longwavelength description of the system, the nonlocal dependence on the concentration field in Eq. (11) is expanded in gradients as

$$
\begin{aligned}
c\left(\mathbf{r}_{1}+\boldsymbol{\xi}, \hat{\mathbf{u}}_{2}, t\right)= & c\left(\mathbf{r}_{1}, \hat{\mathbf{u}}_{2}\right)+\xi_{\alpha} \partial_{r_{1 \alpha}} c\left(\mathbf{r}_{1}, \hat{\mathbf{u}}_{2}\right) \\
& +\frac{1}{2} \xi_{\alpha} \xi_{\beta} \partial_{r_{1 \alpha}} \partial_{r_{1 \beta}} c\left(\mathbf{r}_{1}, \hat{\mathbf{u}}_{2}\right)+O\left(\nabla^{3}\right),
\end{aligned}
$$

where the expansion has been truncated at second order in the gradients. This amounts to a hydrodynamic description up through Navier-Stokes order in the coarse-grained densities.

Secondly, the balance equations for the polarization and the nematic order parameter couple to higher moments of the probability distribution. To obtain a closed set of equations we need an additional assumption that allows these higher moments to be expressed in terms of the first three moments of the probability distribution. We assume that at long times (times longer than all microscopic diffusion times) the higher moments become functionals of the first three moments [24] and the probability distribution has the form

$$
c(\mathbf{r}, \hat{\mathbf{u}}, t) \rightarrow c\left(\hat{\mathbf{u}},\left[y_{\alpha}(\mathbf{r}, t)\right]\right),
$$

where $y_{\alpha}(\mathbf{r}, t)=\left\{\rho(\mathbf{r}, t), \mathbf{P}(\mathbf{r}, t), Q_{\alpha \beta}(\mathbf{r}, t)\right\}$ and the square brackets denote the fact that the probability distribution $c$ is a functional of these fields. Once such a functional assumption is made, the Smoluchowski equation can be self-consistently solved to obtain the probability distribution in terms of the slow variables in the system and their gradients. In the context of equilibrium systems, this procedure is implemented using slow variables that are hydrodynamic, i.e., they are the conserved quantities of the system. But as has been shown in the context of granular fluids, it can carried out using overdamped variables as in the case at hand as well [25].

Here this procedure is implemented in a much simpler context. It is assumed that the probability distribution is a linear functional of the slow variables and the solution to the Smoluchowski equation is obtained to lowest order in the gradients in the slow variables of interest here. Then, it follows immediately that the probability distribution is given by

$$
c(\mathbf{r}, \hat{\mathbf{u}}, t)=\frac{1}{2 \pi} \rho(\mathbf{r}, t)\left[1+2 \mathbf{P}(\mathbf{r}, t) \cdot \hat{\mathbf{u}}+4 Q_{\alpha \beta}\left(\hat{u}_{\alpha} \hat{u}_{\beta}-\frac{1}{2} \delta_{\alpha \beta}\right)\right] .
$$

Using the two approximations given by Eqs. (20) and (22), a set of closed macroscopic equations for the density, polarization, and nematic order parameter are obtained. Including only diffusion and self-propulsion contributions to the fluxes, these equations are given by

$$
\begin{gathered}
\partial_{t} \rho+v_{0} \boldsymbol{\nabla} \cdot(\rho \mathbf{P})=\frac{3 D_{0}}{4} \nabla^{2} \rho+\frac{D_{0}}{2} \partial_{\alpha} \partial_{\beta}\left(\rho Q_{\alpha \beta}\right), \\
\partial_{t}\left(\rho P_{\alpha}\right)+\frac{1}{2} v_{0} \partial_{\alpha} \rho+v_{0} \partial_{\beta}\left(\rho Q_{\alpha \beta}\right) \\
=-D_{R} \rho P_{\alpha}+\frac{5 D_{0}}{8} \nabla^{2}\left(\rho P_{\alpha}\right)+\frac{D_{0}}{4} \partial_{\alpha} \boldsymbol{\nabla} \cdot(\rho \mathbf{P}), \\
\partial_{t}\left(\rho Q_{\alpha \beta}\right)+\frac{v_{0}}{4}\left[\partial_{\alpha}\left(\rho P_{\beta}\right)+\partial_{\beta}\left(\rho P_{\alpha}\right)-\delta_{\alpha \beta} \boldsymbol{\nabla} \cdot(\rho \mathbf{P})\right] \\
=-4 D_{R} \rho Q_{\alpha \beta}+\frac{D_{0}}{8}\left[\partial_{\alpha} \partial_{\beta}-\frac{1}{2} \delta_{\alpha \beta} \nabla^{2}\right] \rho+\frac{D_{0}}{6}\left[\partial _ { \gamma } \left(\partial_{\alpha} \rho Q_{\beta \gamma}\right.\right. \\
\left.\left.+\partial_{\beta} \rho Q_{\alpha \gamma}-\delta_{\alpha \beta} \partial_{\sigma} \rho Q_{\sigma \gamma}\right)+\frac{7}{2} \nabla^{2} \rho Q_{\alpha \beta}\right] .
\end{gathered}
$$

The excluded volume contributions to the fluxes are given in the Appendix. In Eqs. (23)-(25), self-propulsion generates convective-type terms that couple the density and the nematic order parameter to the polarization, reflecting the fact that self-propulsion yields a mass flux in the direction of polarization. In the following sections, these equations are used to study the possible steady state of the systems and their stability.

\section{A. Homogeneous states}

The bulk states of the system are determined by the solutions of the homogeneous hydrodynamic equations. Dropping all gradients terms, but including excluded volume effects, these are given by

$$
\partial_{t} \rho=0,
$$




$$
\begin{gathered}
\partial_{t} \rho P_{\alpha}=-D_{R} \rho P_{\alpha}+\frac{4}{3} D_{R} \eta_{0} \rho^{2} P_{\beta} Q_{\alpha \beta}, \\
\partial_{t} \rho Q_{\alpha \beta}=-4 D_{R} \rho\left(1-\eta_{0} \rho / 3\right) Q_{\alpha \beta},
\end{gathered}
$$

where $\eta_{0}=2 \ell^{2} / \pi$ is the excluded volume of a rod. As anticipated, the self-propulsion velocity $v_{0}$ does not enter the homogeneous equations. This is an artifact of our modeling of self-propulsion solely as a center of mass force and hence an effective center of mass velocity in the overdamped limit in the microscopic model considered here. The homogeneous equations are therefore identical to those of an equilibrium collection of overdamped hard rods. At low density the system forms an isotropic liquid state, with $\rho=\rho_{0}, \mathbf{P}=0$, and $Q_{\alpha \beta}=0$. The isotropic state becomes unstable for $\rho_{0}>\rho_{N}$ $=3 / \eta_{0}=3 \pi / 2 \ell^{2}$ where the coefficient of $Q_{\alpha \beta}$ on the righthand side of Eq. (28) changes sign. For $\rho_{0}>\rho_{N}$ a collection of hard rods forms a nematic liquid crystal, characterized by broken orientational symmetry along the direction of a unit vector $\hat{\mathbf{n}}$ known as the director. The alignment tensor takes the form

$$
Q_{\alpha \beta}=S\left(\hat{n}_{\alpha} \hat{n}_{\beta}-\frac{1}{2} \delta_{\alpha \beta}\right)
$$

This is the well-known result due to Onsager. In mean-field theory the isotropic-nematic transition is continuous in two dimensions [26]. To obtain the value $S$ of the order parameter one must retain terms cubic in $Q_{\alpha \beta}$ in Eq. (28) which arise from higher order correlations neglected here. These yield a term $\sim w \rho_{0}^{3} Q_{\gamma \delta} Q_{\gamma \delta} Q_{\alpha \beta}$, where in the present context $w$ is a phenomenological parameter, independent of density. One then finds $S=\frac{1}{\rho_{0}} \sqrt{\frac{8}{w}\left(\rho_{0} / \rho_{N}-1\right)}$ in the nematic state. Finally, there is no homogeneous polarized state.

\section{B. Fluctuations in the isotropic state}

In this section we examine the dynamics of fluctuations in the isotropic state for $\rho_{0}<\rho_{N}$ to study its stability. The only hydrodynamic variable in the isotropic state is the concentration of filaments, $\rho$. Fluctuations in both the polarization and the alignment tensor decay on microscopic time scales. However, while fluctuations in the alignment tensor couple to the density to higher order in the gradients and can be safely neglected, polarization fluctuations can qualitatively change the nature of the hydrodynamic modes at intermediate length scales. We therefore consider the coupled dynamics of fluctuations of the density $\delta \rho(\mathbf{r}, t)=\rho(\mathbf{r}, t)-\rho_{0}$ and polarization $\delta \mathbf{P}(\mathbf{r}, t)=\mathbf{P}(\mathbf{r}, t)$ from their homogeneous values $\rho=\rho_{0}$ and $\mathbf{P}=0$. It is convenient to expand the fluctuations in Fourier components

$$
\delta \widetilde{y}(\mathbf{k}, t)=\int d \mathbf{r} e^{i \mathbf{k} \cdot \mathbf{r}} \delta y(\mathbf{r}, t)
$$

and to write the polarization in terms of its components longitudinal and transverse to $\hat{\mathbf{k}}$, as $\widetilde{\mathbf{P}}(\mathbf{k}, t)=\hat{\mathbf{k}} \delta P_{\|}(\mathbf{k}, t)$ $+\hat{\mathbf{k}}_{\perp} \delta P_{\perp}(\mathbf{k}, t)$, where $\hat{\mathbf{k}}=\mathbf{k} /|\mathbf{k}|$ and $\hat{\mathbf{k}}_{\perp}=\hat{z} \times \hat{\mathbf{k}}$ are unit vectors longitudinal and perpendicular to $\mathbf{k}$. The transverse compo- nent of the polarization decouples from the density and will be neglected below. The linearized coupled equations for fluctuations in the density and longitudinal polarization, which corresponds to splay deformations of the polarization field, are given by

$$
\begin{gathered}
\partial_{t} \delta \widetilde{\rho}=-D k^{2} \delta \widetilde{\rho}+i k v_{0} \rho_{0} \delta \widetilde{P}_{\|} \\
\partial_{t} \delta \widetilde{P}_{\|}=-\left[D_{R}+D_{p} k^{2}\right] \delta \widetilde{P}_{\|}+i k v_{0} \frac{\delta \widetilde{\rho}}{2 \rho_{0}},
\end{gathered}
$$

where in the low density approximation considered here,

$$
\begin{gathered}
D=\frac{3 D_{0}}{4}\left[1+\frac{\rho_{0}}{\rho_{N}}\right], \\
D_{p}=\frac{7}{8} D_{0} .
\end{gathered}
$$

Note that the splay diffusion constant $D_{p}$ can naturally be written as $D_{p}=K_{1} / \zeta$, with $K_{1}$ the splay elastic constant and $\zeta=\frac{k_{B} T}{D_{0}}$ a friction coefficient. At low density we obtain $K_{1}$ $=\frac{7}{8} k_{B} T$.

If $v_{0}=0$, Eqs. (31) and (32) decouple: density fluctuations decay via diffusion and polarization fluctuations are overdamped with decay rate $D_{R}$. Conversely, at finite $v_{0}$, for $t$ $\gg D_{R}^{-1}$ and long wavelengths, we can assume that polarization fluctuations relax quickly to a value determined by the inhomogeneous density field $\delta \widetilde{P}_{\|} \simeq i k v_{0} \delta \tilde{\rho} /\left(2 D_{R} \rho_{0}\right)$, which yields traveling density waves of speed $v_{0}$.

In general we look for wavelike solutions with time dependence given as linear combinations of terms of the form $\sim e^{\lambda_{\nu}(k) t}$, where $\lambda_{\nu}(k)$ are the dispersion relations of the hydrodynamic modes of the system. The modes controlling the decay of density and splay fluctuations in the isotropic state are given by

$$
\lambda_{1,2}=-\frac{D_{R}+\left(D_{p}+D\right) k^{2}}{2} \pm \frac{1}{2} \sqrt{\left[D_{R}+\left(D_{p}-D\right) k^{2}\right]^{2}-2 v_{0}^{2} k^{2}} .
$$

The sign of the real part of the eigenvalues $\lambda_{\nu}(k)$ controls the linear stability of the homogeneous state. It is easy to see that in the isotropic state $\operatorname{Re}\left[\lambda_{\nu}(k)\right]<0$ for $\nu=1,2$ and all values of parameters, indicating that the isotropic state is linearly stable for all $\rho_{0}<\rho_{N}$. The nature of the modes changes, however, from diffusive to propagating when the argument of the square root on the right-hand side of Eq. (35) changes sign. At low density $D_{p}>D$ and the system exhibits propagating density waves for $v_{0} \geqslant v_{0}(k)$, with

$$
v_{0}(k)=\frac{D_{R}+\left(D_{p}-D\right) k^{2}}{\sqrt{2} k} .
$$

The crossover from diffusive to propagating density fluctuations is displayed qualitatively in Fig. 2. There is a lower value $v_{c 0}=\sqrt{2 D_{R}\left(D_{p}-D\right)} \sim \sqrt{D_{R} D_{0}} / 2$ of $v_{0}$ below which the behavior is always diffusive. For a fixed value of $v_{0}>v_{c 0}$ propagating waves exist in a range of wave vector $k_{c 1} \leqslant k$ $\leqslant k_{c 2}$, with 


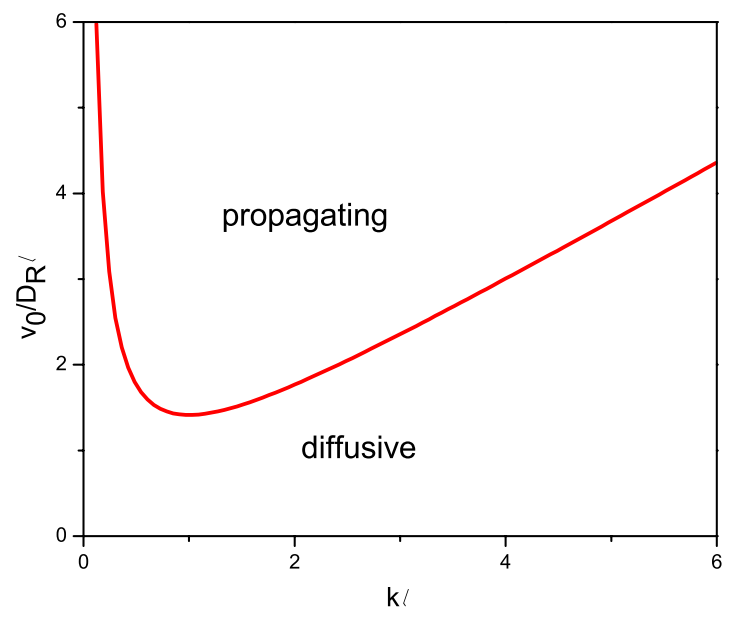

FIG. 2. (Color online) A description of the crossover from diffusive to propagating density fluctuations in the isotropic state. The solid line (red online) is the boundary $v_{0}(k)$ given in Eq. (36) for $\frac{D_{p}-D}{\ell D_{R}}=1$. Velocities on the vertical axis are measured in units of $\ell D_{R}$, where $\ell$ is the length of the rods. The magnitude of the wave vector $k$ is measured in units of $\ell^{-1}$. The boundary $v_{0}(k)$ has a minimum at $v_{c 0}$ below which the density fluctuations are always diffusive. For $v_{0}>v_{c 0}$, the system can support propagating sound waves when $k_{c 1}\left(v_{0}\right) \leqslant k \leqslant k_{c 2}\left(v_{0}\right)$, with $k_{c i}\left(v_{0}\right)$ given in Eq. (37).

$$
k_{c 1, c 2}=\frac{v_{0}}{D_{p}-D}\left(1 \mp \sqrt{1-\frac{v_{c 0}^{2}}{2 v_{0}^{2}}}\right) .
$$

This range widens as $v_{0}$ increases, with $\lim _{v_{0} \rightarrow \infty} k_{c 1}=0$ and $\lim _{v_{0} \rightarrow \infty} k_{c 2}=\infty$. This behavior resembles closely the appearance of propagating sound waves at intermediate wave vectors in a compressible fluid that interacts frictionally with a substrate [28]. The polarization here plays the same role as the flow velocity in the Navier-Stokes equations of $\operatorname{Re}[28]$ and the self-propulsion effectively lowers the damping of polarization fluctuations, yielding propagating waves. Finally, the appearance of propagating waves in active systems of the type considered here was also recently predicted by Ramaswamy using a phenomenological approach [29].

Using $D_{0} \sim \ell^{2} D_{R}$, we obtain $v_{c 0} \sim D_{0} / \ell \sim \ell D_{R}$. It is useful to introduce two characteristic length scales as

$$
\begin{gathered}
\ell_{d i f f}=\sqrt{\frac{2 D}{D_{R}}}, \\
\ell_{s p}=\frac{v_{0}}{D_{R}},
\end{gathered}
$$

where $\ell_{\text {diff }}$ and $\ell_{s p}$ are the distances traveled by the center of mass of a rod due to diffusion and self-propulsion, respectively, during the characteristic time scale for rotational diffusion $D_{R}^{-1}$. The condition $v_{0} \gg v_{c 0}$ of large self-propulsion corresponds to $\ell_{s p} \gg \ell_{\text {diff. }}$. In this limit

$$
k_{c 1} \simeq \frac{1}{\sqrt{2}} \frac{1}{\ell_{s p}}+\frac{2 \sqrt{2}}{\ell_{\text {diff }}}\left(\frac{\ell_{s p}}{\ell_{\text {diff }}}\right),
$$

$$
k_{c 2} \simeq \frac{1}{\ell_{s p}} .
$$

The modes are propagating for all length scales larger than $\ell_{\text {diff }}^{2} / \ell_{s p} \ll \ell_{\text {diff }}$ and smaller than $\ell_{s p}$. For any fixed value of $v_{0}$, the modes always become diffusive when $k \rightarrow 0$. Finally, at high density we expect $D>D_{p}$. In this case propagating waves will exist in a range of wave vector for all nonzero values of $v_{0}$. Furthermore, increasing the density will result to a strong suppression of rotational diffusion from entanglement. In this case propagating waves will exist for essentially all length scales.

\section{Fluctuations in the nematic state}

Here we examine the linear stability of the nematic state, characterized by a uniform density $\rho_{0}>\rho_{N}$, alignment tensor $Q_{\alpha \beta}^{0}=S\left(\hat{n}_{0 \alpha} \hat{n}_{0 \beta}-\frac{1}{2} \delta_{\alpha \beta}\right)$, and $\mathbf{P}=0$. The ordered state is symmetric for $\hat{\mathbf{n}}_{0} \rightarrow-\hat{\mathbf{n}}_{0}$. The hydrodynamic variables in this case are the density and the director. As for the isotropic state, fluctuations in the polarization, although overdamped, can qualitatively change the dynamics of the system and ultimately render the uniform nematic state unstable. They will therefore be incorporated in the analysis below. We assume the system to be deep in the nematic state and neglect fluctuations in the magnitude $S$ of the order parameter. We consider small fluctuations about the ordered state by letting

$$
\begin{gathered}
\rho(\mathbf{r}, t)=\rho_{0}+\delta \rho(\mathbf{r}, t), \\
\mathbf{P}(\mathbf{r}, t)=\delta \mathbf{P}(\mathbf{r}, t), \\
Q_{\alpha \beta}(\mathbf{r}, t)=Q_{\alpha \beta}^{0}+S\left[\hat{n}_{0 \alpha} \delta n_{\perp \beta}(\mathbf{r}, t)+\delta n_{\perp \alpha}(\mathbf{r}, t) \hat{n}_{0 \beta}\right],
\end{gathered}
$$

where $\delta \mathbf{n}_{\perp}$ denotes fluctuations perpendicular to $\hat{\mathbf{n}}_{0}$. To linear order, this is the only fluctuation in the director field that preserves $|\hat{\mathbf{n}}|=1$. It is convenient to choose a coordinate system with the $x$ axis along $\hat{\mathbf{n}}_{0}$, so that $\delta \mathbf{n}_{\perp}=\delta n_{y} \hat{\mathbf{y}}$.

The fluctuations $\delta \mathbf{P}$ in the polarization are overdamped at the rate $D_{R}$. For $t \gg D_{R}^{-1}$ we neglect $\partial_{t} \delta \mathbf{P}$ and eliminate polarization fluctuations in favor of the density and director fields. The resulting hydrodynamic equations are given by

$$
\begin{gathered}
\partial_{t} \delta \rho=\left(D_{x} \partial_{x}^{2}+D_{\perp} \nabla_{\perp}^{2}\right) \delta \rho+D_{0} S \rho_{0}(1+2 \alpha) \partial_{x} \boldsymbol{\nabla}_{\perp} \cdot \delta \mathbf{n}_{\perp}, \\
\partial \delta \mathbf{n}_{\perp}=\left(K_{3} \partial_{x}^{2}+K_{1} \nabla_{\perp} \nabla_{\perp} \cdot\right) \delta \mathbf{n}_{\perp}+\frac{D_{0}}{8 S}(3+2 \alpha) \partial_{x} \nabla_{\perp} \frac{\delta \rho}{\rho_{0}},
\end{gathered}
$$

with

$$
\begin{aligned}
& D_{x}=\frac{D_{0}}{4}[3+S+2 \alpha(1+S)], \\
& D_{\perp}=\frac{D_{0}}{4}[3-S+2 \alpha(1-S)],
\end{aligned}
$$




$$
K_{1}=K_{3}=\frac{D_{0}}{4}(3+\alpha)
$$

and $\alpha=\frac{v_{0}^{2}}{D_{R} D} \sim \frac{v_{0}^{2}}{4 v_{c 0}^{2}}$ a dimensionless parameter. As expected on the basis of symmetry, only the square of the self-propulsion velocity enters the equations for the nematic. Self-propulsion enhances diffusion along the direction $x$ of alignment. It also stiffens both the bend and splay elastic constants $K_{3}$ and $K_{1}$. To analyze the stability of the nematic state we expand the fluctuations in Fourier modes at wave vector $\mathbf{k}$. Denoting by $\phi$ the angle that $\mathbf{k}$ makes with $\hat{\mathbf{n}}_{0}$, the equations for the Fourier amplitudes of the fluctuations are

$$
\begin{gathered}
\partial_{t} \delta \widetilde{\rho}=-D_{\rho \rho}(\alpha, \phi) k^{2} \delta \widetilde{\rho}-S \rho_{0} D_{\rho n}(\alpha, \phi) k^{2} \delta \widetilde{n}_{y}, \\
\partial_{t} \delta \tilde{n}_{y}=-D_{n \rho}(\alpha, \phi) k^{2} \frac{\delta \widetilde{\rho}}{S \rho_{0}}-D_{n n}(\alpha) k^{2} \delta \tilde{n}_{y},
\end{gathered}
$$

with

$$
\begin{gathered}
D_{\rho \rho}=\left(D_{x} \sin ^{2} \phi+D_{\perp} \cos ^{2} \phi\right), \\
D_{n n}=\frac{D_{0}}{4}(3+\alpha), \\
D_{\rho n}=D_{0}(1+2 \alpha) \cos \phi \sin \phi, \\
D_{n \rho}=\frac{D_{0}}{8}(3+2 \alpha) \cos \phi \sin \phi .
\end{gathered}
$$

When $\phi=0$, i.e., for wave vectors $\mathbf{k}$ parallel to $\hat{\mathbf{n}}_{0}$, density and director fluctuations decouple and the modes are always stable. To linear order, this geometry corresponds to pure bend fluctuations of the director. The modes also decouple for $\phi=\pi / 2$, corresponding to $\mathbf{k}$ normal to the direction of broken symmetry. To analyze the linear stability of the nematic state for arbitrary angles $\phi$ we must examine the dispersion relations of the hydrodynamic modes associated with Eqs. (47) and (48). These are easily obtained as

$$
\lambda_{ \pm}=\frac{k^{2}}{2}\left[-\left(D_{\rho \rho}+D_{n n}\right) \pm \sqrt{\left(D_{\rho \rho}-D_{n n}\right)^{2}+4 D_{\rho n} D_{n \rho}}\right] .
$$

The eigenvalues are always real, corresponding to diffusive modes. The mode $\lambda_{-}$is always negative, indicating stable decay of fluctuations. The mode $\lambda_{+}$changes sign for

$$
D_{\rho n} D_{n \rho}>D_{\rho \rho} D_{n n} \text {. }
$$

It is easy to verify that this condition can never be satisfied when $v_{0}=0$, i.e., the nematic state is stable in the absence of self-propulsion. On the other hand, the homogeneous nematic state becomes unstable for all values of $k$ at large enough $v_{0}$. The condition for the onset of the instability obtained from Eq. (54) yields a boundary $v_{c}(\phi, S)$. The nematic state is unstable for $v_{0}>v_{c}(\phi, S)$, as shown in Fig. 3. For a fixed value of $S$, there is a minimum threshold value $v_{c}(S)$ required to destabilize the nematic state. For a fixed value of $v_{0}$ $>v_{c}(S)$ the nematic state is unstable for all spatial gradients

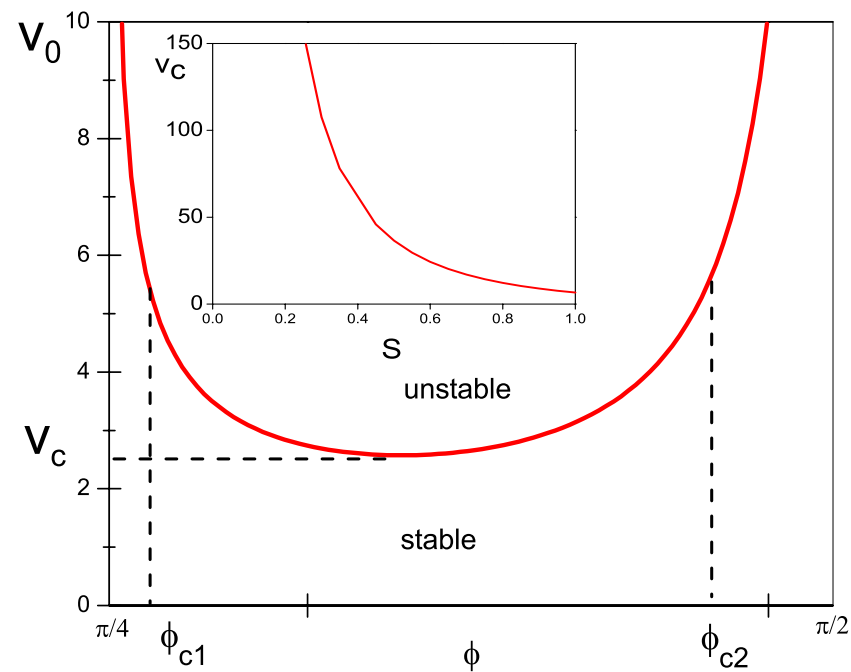

FIG. 3. (Color online) The boundary $v_{c}(\phi, S)$ of the stability of the homogeneous nematic state is shown as a function of the angle $\phi$ between the wave vector and the direction of nematic order for $S=1$. The nematic state is always stable for all $\phi$ when $v_{0}<v_{c}(S)$. For a fixed value of $v_{0}>v_{c}(S)$ the nematic state is unstable for $\phi_{c 1}(S) \leqslant \phi \leqslant \phi_{c 2}(S)$. The behavior of $v_{c}(S)$ with $S$ is shown in the inset. The critical speed $v_{c}(S)$ diverges as $S \rightarrow 0$.

such that $\phi_{c 1} \leqslant \phi \leqslant \phi_{c 2}$. For $S=1$, we find $\lim _{v_{0} \rightarrow \infty} \phi_{c 1}=\frac{\pi}{4}$ and $\lim _{v_{0} \rightarrow \infty} \phi_{c 2}=\frac{\pi}{2}$, and the system becomes unstable to all spatial gradients such that the splay wins over bend. Finally, as illustrated in Fig. 4 and the inset in Fig. 3, the unstable region shrinks in size as the magnitude of nematic order in the system decreases.

The instability of the nematic state arises from a subtle interplay of splay and bend deformations and diffusion longitudinal and transverse to the direction of nematic order. It

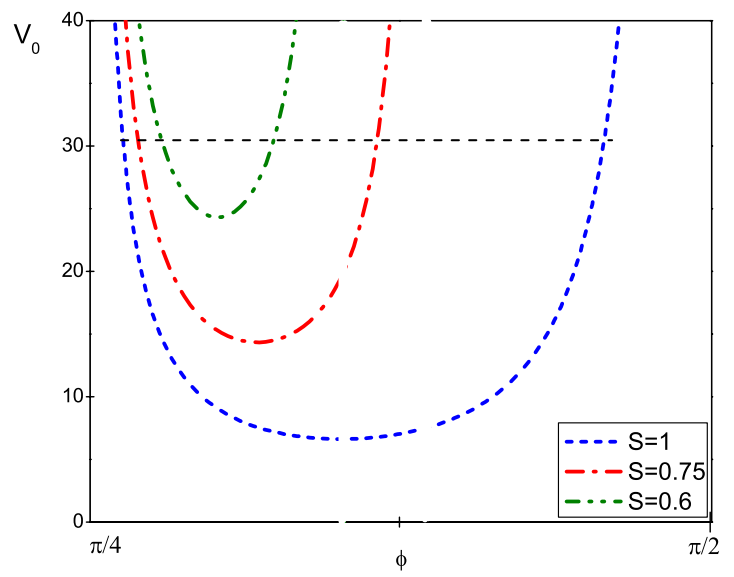

FIG. 4. (Color online) The boundary $v_{c}(\phi, S)$ above which the homogeneous nematic state is linearly unstable is shown as a function of the angle $\phi$ between the wave vector and the direction of nematic order for three values of $S$. The horizontal line at a fixed $v_{0}$ intersects each curve at two values of $\phi, \phi_{c 1}(S)<\phi_{c 2}(S)$. The nematic state is unstable for $\phi_{c 1}(S) \leqslant \phi \leqslant \phi_{c 2}(S)$. The figure illustrates that the range of angles $\phi$ for which the system becomes unstable decreases as the degree of order in the system decreases. 
only occurs for angles $\phi>\pi / 4$, corresponding to situations where splay deformations of the nematic director exceed bend deformations. It does not, however, occur for pure splay $(\phi=\pi / 2)$, other than at $v_{0} \rightarrow \infty$. In a nematic, curvature inhomogeneities of the director yield mass currents, with splay deformations $\sim \partial_{y} \delta n_{y}$ yielding current along the direction $x$ of mean order and bend deformations $\sim \partial_{x} \delta n_{y}$ yielding current along the $y$ direction. Self-propulsion enhances both contributions. In addition, it enhances mass diffusion both longitudinal and transverse to the direction of order, as indicated in Eqs. (44) and (45). However, while the enhancement of $D_{x}$ from self-propulsion grows as the nematic order increases and is maximum at $S=1$, the enhancement of $D_{\perp}$ drops as one moves deeper into the nematic state and vanishes when $S=1$. Thus, if a curvature in the director field induces a mass flux that requires relaxation through appreciable diffusion perpendicular to the mean director, then, beyond the threshold value $v_{c}$, the system becomes unstable. A fluctuation in the director field corresponding to a large splay and a small bend results in exactly such mass fluxes. Conversely, when bend exceeds splay longitudinal diffusion is the main mechanism that restores orientational order. This is enhanced by self-propulsion and the system remains linearly stable.

The analysis described above only includes terms up to quadratic order in the gradients and predicts that the nematic state is unstable on all length scales. We expect that terms of higher order in the gradients arising from excluded volume and diffusion currents will stabilize the nematic state at large wave vectors [27], setting a length scale for the typical size of the ordered regions, not unlike what has been observed in the simulations by Peruani et al. [15].

\section{SUMMARY AND DISCUSSION}

In this paper, we have discussed the collective dynamics of self-propelled hard rods on a frictional substrate. The rods interact through excluded volume interactions. The selfpropulsion was implemented as a center of mass force acting on each rod that propels it along its long axis. Selfpropulsion breaks the nematic symmetry at the level of the microscopic equations of motion, but since it yields only a center of mass force on each rod it is insufficient to generate a macroscopic polarized state. The existence of a polar state requires either microscopic torques that turn the particle towards an externally determined direction (for instance as seen in the case of chemotaxis in bacterial motion) or an aligning interaction that can distinguish the two ends of the extended object, which a physical excluded volume interaction of rods does not. As a result, the only possible bulk states of the self-propelled systems are those possible in equilibrium: isotropic and nematic.

Self-propulsion, does, however have a profound effect on the nature of the fluctuations in each of these states. In the isotropic state self-propulsion yields the appearance of propagating waves in a range of wave vectors, as shown in Fig. 2. This result is closely analogous to the appearance of sound waves in a compressible fluid on a frictional substrate, predicted many years ago by Ramaswamy and Mazenko
[28]. This phenomenon has not been observed in equilibrium systems where it requires a very small value of the friction. In self-propelled systems, self-propulsion itself effectively lowers the friction and should yield a wide range of parameters where propagating density waves may be observable. Propagating density waves may indeed have been seen in the collective dynamics of epithelial cells on a substrate [17]. In the nematic state fluctuations in the local polarization yield mass fluxes and can destabilize the homogeneous nematic state. This has been previously observed in the numerical study of [15]. Further, before the onset of the instability, these same director fluctuations and the resulting anomalous mass flux associated with them account for the large number of fluctuations predicted in [10]. In fact, Eqs. (42) and (43) for fluctuations in the nematic state are identical to those considered in [10], although with microscopic (low density) values for all the parameters.

In closing, we would like to note that our work has several limitations. First, we neglect all correlations in deriving the Smoluchowski equation and in the functional assumption made to obtain the hydrodynamic equations. On the other hand, our hydrodynamic equations have precisely the structure predicted on the basis of pure symmetry considerations. This suggests that the behavior obtained here may be generic. Therefore, further insight into the transport processes can be obtained from numerical simulation of the model system and comparing the results to the inherently low density theory presented here. Secondly, the overdamped Langevin microdynamics that describes the hard core interaction as an equilibrium mean-field excluded volume effect may not be an adequate starting point to incorporate self-propulsion. It can be shown that the momentum transfer that occurs in the interactions between self-propelled hard rods modifies the diffusion processes in the system [23]. These effects can be captured by starting with the true Langevin equations describing a system of hard rods on a substrate and then obtaining the associated Fokker-Planck equation, as will be described in a future publication [23].

\section{ACKNOWLEDGMENTS}

Research supported by the NSF through Grant Nos. DMR-0305407 and DMR-0705105. M.C.M. also received support from the Institut Curie in Paris via a RotschildYvette-Mayent grant. She thanks both the Institut Curie and ESPCI for their hospitality during the completion of some of this work. Finally, we thank Sriram Ramaswamy for useful discussions and for alerting us to Ref. [28].

\section{APPENDIX: HYDRODYNAMIC FLUXES}

In this Appendix, we give the expression for the excluded volume contributions to the translational and rotational fluxes defined in Eqs. (18) and (19). These have been obtained assuming a low moments closure for the one-particle distribution function of the form given in Eq. (21). The excluded volume contributions to the translational fluxes are given by 


$$
\begin{aligned}
& J_{\alpha}^{e x}=D_{0} \eta_{0} \partial_{\beta}\left[-\frac{1}{2} \rho^{2}\left(Q_{\alpha \beta}+\frac{3}{4} \delta_{\alpha \beta}\right)+\frac{2}{9} \rho^{2} Q_{\alpha \beta}^{2}\right. \\
& \left.+\frac{7}{18} \rho^{2} \delta_{\alpha \beta} T r Q^{2}\right]+\frac{2}{3} D_{0} \eta_{0} \rho \partial_{\beta} \rho Q_{\alpha \beta}, \\
J_{\alpha \beta}^{e x}= & -\frac{1}{8} D_{0} \eta_{0}\left(\Delta_{\alpha \beta \gamma \sigma}+4 \delta_{\alpha \sigma} \delta_{\beta \gamma}\right) \rho P_{\gamma} \partial_{\sigma} \rho+\frac{1}{18} D_{0} \eta_{0}\left(\Delta_{\alpha \beta \omega \gamma}\right. \\
& \left.+6 \delta_{\alpha \omega} \delta_{\beta \gamma}\right) \rho P_{\sigma} \partial_{\omega} \rho Q_{\sigma \gamma}+\frac{1}{18} D_{0} \eta_{0}\left[\left(\Delta_{\alpha \beta \omega \gamma}\right.\right. \\
& \left.\left.-\delta_{\alpha \beta} \delta_{\omega \gamma}\right) \rho P_{\omega} \partial_{\sigma} \rho Q_{\sigma \gamma}+\rho P_{\omega} \partial_{\omega} \rho Q_{\alpha \beta}\right] \\
J_{\alpha \beta \gamma}^{e x}= & \frac{1}{12} D_{0} \eta_{0}\left\{-\frac{3}{8}\left(\delta_{\alpha \gamma} \delta_{\beta \sigma}+\delta_{\alpha \sigma} \delta_{\beta \gamma}-\delta_{\alpha \beta} \delta_{\gamma \sigma}\right) \partial_{\sigma} \rho^{2}\right. \\
& +\frac{1}{3} \rho \partial_{\sigma} \rho\left(7 \delta_{\gamma \sigma} Q_{\alpha \beta}+\delta_{\alpha \gamma} Q_{\beta \sigma}+\delta_{\beta \sigma} Q_{\alpha \gamma}+\delta_{\beta \gamma} Q_{\alpha \sigma}\right. \\
& \left.+\delta_{\alpha \sigma} Q_{\beta \gamma}-2 \delta_{\alpha \beta} Q_{\gamma \sigma}\right)-\rho\left(7 \delta_{\gamma \sigma} Q_{\alpha \beta}+\delta_{\alpha \gamma} Q_{\beta \sigma}\right. \\
& \left.+\delta_{\beta \sigma} Q_{\alpha \gamma}+\delta_{\beta \gamma} Q_{\alpha \sigma}+\delta_{\alpha \sigma} Q_{\beta \gamma}-2 \delta_{\alpha \beta} Q_{\gamma \sigma}\right) \partial_{\sigma} \rho \\
& +\frac{1}{3} \partial_{\sigma} \rho^{2}\left(Q_{\alpha \beta} Q_{\gamma \sigma}+Q_{\alpha \gamma} Q_{\beta \sigma}+Q_{\alpha \sigma} Q_{\gamma \beta}-\delta_{\alpha \beta} Q_{\gamma \omega} Q_{\sigma \omega}\right) \\
+ & \rho^{2}\left(\delta_{\alpha \sigma} Q_{\beta \omega}+\delta_{\beta \sigma} Q_{\alpha \omega}-\delta_{\alpha \beta} Q_{\sigma \omega}\right) Q_{\gamma \omega}+\rho^{2}\left(\delta_{\alpha \gamma} Q_{\beta \omega}\right.
\end{aligned}
$$

$$
\begin{aligned}
& \left.+\delta_{\beta \gamma} Q_{\alpha \omega}-\delta_{\alpha \beta} Q_{\gamma \omega}\right) Q_{\sigma \omega}+\frac{1}{4} \rho^{2}\left(\delta_{\alpha \gamma} \delta_{\beta \sigma}+\delta_{\alpha \sigma} \delta_{\beta \gamma}\right. \\
& \left.\left.-19 \delta_{\alpha \beta} \delta_{\gamma \sigma}\right) \operatorname{Tr}\left(Q^{2}\right)+9 \rho^{2} \delta_{\gamma \sigma} Q_{\alpha \beta}^{2}\right\},
\end{aligned}
$$

where the notation

$$
\Delta_{\alpha \beta \gamma \sigma}=\delta_{\alpha \beta} \delta_{\gamma \sigma}+\delta_{\alpha \gamma} \delta_{\beta \sigma}+\delta_{\alpha \sigma} \delta_{\beta \gamma}
$$

has been introduced for compactness. The excluded volume contributions to the rotational fluxes are

$$
\begin{aligned}
R_{\alpha}^{e x}= & -\frac{1}{3} D_{R} \eta_{0}\left[4 \rho^{2} P_{\beta} Q_{\alpha \beta}+\frac{l^{2}}{6} \rho P_{\alpha} \nabla^{2} \rho-\frac{l^{2}}{3} \rho P_{\beta} \partial_{\alpha} \partial_{\beta} \rho\right. \\
& \left.+\frac{l^{2}}{18} \rho P_{\beta} \nabla^{2}\left(\rho Q_{\alpha \beta}\right)\right]-D_{R} \eta_{0} \frac{l^{2}}{27}\left[\rho P_{\gamma} \partial_{\gamma} \partial_{\beta}\left(\rho Q_{\alpha \beta}\right)\right. \\
& \left.+\left(\rho P_{\beta} \partial_{\alpha} \partial_{\gamma}-\rho P_{\alpha} \partial_{\beta} \partial_{\gamma}\right)\left(\rho Q_{\beta \gamma}\right)\right], \\
R_{\alpha \beta}^{e x}= & -\frac{4}{3} D_{R} \eta_{0} \rho^{2} Q_{\alpha \beta}-\frac{1}{288} D_{R} l^{2} \eta_{0} \rho\left(\partial_{\alpha} \partial_{\beta} \rho-\frac{1}{2} \delta_{\alpha \beta} \nabla^{2} \rho\right) \\
& -\frac{1}{288} D_{R} l^{2} \eta_{0} \rho\left(\delta_{\alpha \sigma} Q_{\beta \gamma}+\delta_{\beta \sigma} Q_{\alpha \gamma}-\delta_{\alpha \beta} Q_{\gamma \sigma}\right) \partial_{\gamma} \partial_{\sigma} \rho .
\end{aligned}
$$

[1] M. C. Cross and P. C. Hohenberg, Rev. Mod. Phys. 65, 851 (1993).

[2] T. Vicsek, A. Czirok, E. Ben-Jacob, I. Cohen, and O. Shochet, Phys. Rev. Lett. 75, 1226 (1995).

[3] J. Toner, Y. Yu, and S. Ramaswamy, Ann. Phys. 318, 170 (2005).

[4] I. S. Aranson, D. Volfson, and L. S. Tsimring, Phys. Rev. E 75, 051301 (2007).

[5] P. M. Chaikin and T. C. Lubensky, Principles of Condensed Matter Physics (Cambridge, New York, 1995).

[6] S. R. de Groot and P. Mazur, Nonequilibrium Thermodynamics (Interscience Publishers, New York, 1962).

[7] N. D. Mermin and H. Wagner, Phys. Rev. Lett. 17, 1133 (1966).

[8] G. Gregoire and H. Chate, Phys. Rev. Lett. 92, 025702 (2004).

[9] R. A. Simha and S. Ramaswamy, Phys. Rev. Lett. 89, 058101 (2002).

[10] S. Ramaswamy, R. A. Simha, and J. Toner, Europhys. Lett. 62, 196 (2003).

[11] V. Narayan, N. Menon, and S. Ramaswamy, J. Stat. Mech.: Theory Exp., 2006, P01005 (2006); V. Narayan, Sriram Ramaswamy, and Narayanan Menon, Science 317, 105 (2007).

[12] C. R. Kube and H. Zhang, Adapt. Behav. 2, 189 (1993).

[13] E. Bertin, M. Droz, and G. Gregoire, Phys. Rev. E 74, 022101 (2006).

[14] I. S. Aranson and L. S. Tsimring, Phys. Rev. E 71, 050901(R) (2005).
[15] F. Peruani, A. Deutsch, and M. Bar, Phys. Rev. E 74, 030904(R) (2006).

[16] Preliminary results suggest, however, that an inhomogeneous polar state can be obtained in a confined geometry.

[17] T. Angelini, M. Marquez, and D. Weitz, March 2007 Meeting of The American Physical Society, Denver, CO, 2007.

[18] H. Chate, F. Ginelli, and R. Montagne, Phys. Rev. Lett. 96, 180602 (2006).

[19] S. Mishra and S. Ramaswamy, Phys. Rev. Lett. 97, 090602 (2006).

[20] O. A. Igoshin, R. Welch, and D. Kaiser, Proc. Natl. Acad. Sci. U.S.A. 101, 4256 (2004).

[21] R. Kemkemer, D. Kling, D. Kaufmann, and H. Gruler, Eur. Phys. J. E 1, 215 (2000).

[22] C. W. Reynolds, ACM SIGGRAPH Computer Graphics 21, 25 (1987), http://portal.acm.org/citation.cfm?id=37402.37406

[23] A. Baskaran and M. C. Marchetti (unpublished).

[24] J. A. McLennan, Introduction to Nonequilibrium Statistical Mechanics (Prentice-Hall, New Jersey, 1989).

[25] J. J. Brey, J. W. Dufty, C. S. Kim, and A. Santos, Phys. Rev. E 58, 4638 (1998).

[26] M. Doi and S. F. Edwards, The Theory of Polymer Dynamics (Oxford University Press, New York, 1986).

[27] A. Ahmadi, M. C. Marchetti, and T. B. Liverpool, Phys. Rev. E 74, 061913 (2006).

[28] S. Ramaswamy and G. F. Mazenko, Phys. Rev. A 26, 1735 (1982).

[29] S. Ramaswamy (unpublished). 